\title{
Chiral dynamics of many-pion systems.
}

\author{
N. N. Achasov * and A. A. Kozhevnikov ${ }^{\dagger}$ \\ Laboratory of Theoretical Physics, \\ Sobolev Institute for Mathematics \\ 630090, Novosibirsk-90, Russia
}

(July 6, 2021)

\begin{abstract}
Based on the Weinberg lagrangian or, in a new language, the lagrangian of hidden local symmetry added with the term induced by the anomalous lagrangian of Wess and Zumino, the dynamics of the decays $\rho \rightarrow 4 \pi$ and $\omega \rightarrow$ $5 \pi$ is considered. The excitation curves of $\rho$ resonance in its decay to $4 \pi$ are obtained for the first time. The comparison with recent CMD-2 data is given. The partial widths of the decays $\omega \rightarrow 2 \pi^{+} 2 \pi^{-} \pi^{0}$ and $\omega \rightarrow \pi^{+} \pi^{-} 3 \pi^{0}$ which can be measured on colliders with luminosity $10^{33} \mathrm{~cm}^{-2} \mathrm{~s}^{-1}$ are evaluated for the first time.
\end{abstract}

11.30.Rd;12.39.Fe;13.30 Eg

Typeset using REVTEX

*Electronic address: achasov@math.nsc.ru

$\dagger$ Electronic address: kozhev@math.nsc.ru 
The decay $\rho \rightarrow 4 \pi$ is a unique source of soft, $|\mathbf{p}| \sim m_{\pi}$, pions, and can be used for the study of the chiral dynamics of many-pion systems. By this reason it attracts much interest [1] [1]. It was found in papers [1] that the above decay should be rather strong, $\mathrm{B}(\rho \rightarrow 4 \pi) \sim 10^{-4}$. The calculations of Ref. [2, 3] were analyzed in detail in the work [4], where a number of shortcomings of the former related with the actual violation of chiral invariance, in particular, the Adler condition for soft pions, was uncovered, The correct result obtained in [4], corresponds to $\mathrm{B}(\rho \rightarrow 4 \pi) \sim 10^{-5}$. The large magnitude of the branching ratio $\mathrm{B}(\rho \rightarrow 4 \pi) \sim 10^{-4}$ obtained in [1] is related, in all appearance, with a very rough method of calculation. A common drawback of all the above cited papers is that their authors evaluate the partial width at the only energy equal to the mass of the $\rho$, as if the latter would be a genuine narrow peak. However, the fact that the width of the $\rho$ resonance is rather large and $\Gamma(\rho \rightarrow 4 \pi, E)$ rises rapidly with the energy increase even at energies inside the $\rho$ peak, push one to think that the magnitude of the $4 \pi$ partial width at the $\rho$ mass cannot be an adequate characteristics of the dynamics of the process. In this respect, it is just the resonance excitation curve in the channel $e^{+} e^{-} \rightarrow \rho^{0} \rightarrow 4 \pi$ is of much interest, being a test ground of various chiral models of the decay under consideration.

Here, based on the Weinberg lagrangian [5] obtained under the nonlinear realization of the chiral symmetry, or, in modern terms, the lagrangian of hidden local symmetry (HLS) [6], the partial widths and resonance excitation curves are calculated for the reactions $e^{+} e^{-} \rightarrow \rho^{0} \rightarrow 2 \pi^{+} 2 \pi^{-}$and $e^{+} e^{-} \rightarrow \rho^{0} \rightarrow \pi^{+} \pi^{-} 2 \pi^{0}$. It is shown that the intensities of the above decays change as fast as two times the phase space variation, upon the energy variation inside the $\rho$ widths. All this means that $e^{+} e^{-}$offers an ideal tool for the study of such effects. The decay widths of charged $\rho$ meson, $\rho^{ \pm} \rightarrow \pi^{ \pm} 3 \pi^{0}$ and $\rho^{ \pm} \rightarrow 2 \pi^{ \pm} \pi^{\mp} \pi^{0}$, as well as $\omega$ meson, $\omega \rightarrow 2 \pi^{+} 2 \pi^{-} \pi^{0} \omega \rightarrow \pi^{+} \pi^{-} 3 \pi^{0}$, are also evaluated.

The HLS approach [6] permits one to include the axial mesons as well [7]. An ideal treatment would consist of that under the assumption of $m_{\rho} \sim E \ll m_{a_{1}}$, the difference between the models with and without $a_{1}$ meson would be reduced to the taking into account the higher derivatives [8] and would be small. In real life one has $m_{a_{1}}^{2}-m_{\rho}^{2} \sim m_{\rho}^{2}$, and the correction may appear to be appreciable even at the $\rho$ mass. In fact, the calculation [泪] shows that the corrections amounts to $\sim 20-30 \%$ in the width. This means, in particular, that the left shoulder of the $\rho$ peak, where the contributions of higher derivatives are vanishing rapidly, is the best place to work. We do not take into account $a_{1}$ meson in the present work. [5]

The amplitudes of the decays of our interest are obtained from the Weinberg lagrangian

$$
\begin{aligned}
\mathcal{L}= & -\frac{1}{4}\left(\partial_{\mu} \boldsymbol{\rho}_{\nu}-\partial_{\nu} \boldsymbol{\rho}_{\mu}+g_{\rho}\left[\boldsymbol{\rho}_{\mu} \times \boldsymbol{\rho}_{\nu}\right]\right)^{2} \\
& +\frac{m_{\rho}^{2}}{2}\left(\boldsymbol{\rho}_{\mu}+\frac{1}{2 g_{\rho} f_{\pi}^{2}} \frac{\left[\boldsymbol{\pi} \times \partial_{\mu} \boldsymbol{\pi}\right]}{1+\boldsymbol{\pi}^{2} / 4 f_{\pi}^{2}}\right)^{2} \\
& +\frac{1}{2}\left(\partial_{\mu} \boldsymbol{\pi}\right)^{2}-\frac{m_{\pi}^{2}}{2} \boldsymbol{\pi}^{2}-\frac{1}{4 f_{\pi}^{2}} \boldsymbol{\pi}^{2}\left(\partial_{\mu} \boldsymbol{\pi}\right)^{2}+\frac{m_{\pi}^{2} \boldsymbol{\pi}^{4}}{8 f_{\pi}^{2}},
\end{aligned}
$$

where $f_{\pi}=92.4 \mathrm{MeV}$ is the pion decay constant, and $g_{\rho}=g_{\rho \pi \pi}$, if one demands the universality condition. Then the so called KSRF relation $2 g_{\rho \pi \pi}^{2} f_{\pi}^{2} / m_{\rho}^{2}=1$ [9] arises, which beautifully agrees with experiment. The $\rho \pi \pi$ coupling constant resulting from this relation 
is $g_{\rho \pi \pi}=5.89$. Introducing the 4 -vector of polarization of the decaying $\rho$ meson, $\varepsilon_{\mu}$, one can write the amplitudes in the form $M=\frac{g_{\rho \pi \pi}}{f_{\pi}^{2}} \varepsilon_{\mu} J_{\mu}$. Let us give the expressions for the current $J_{\mu}$ for all the decay modes considered here.

1) The decay $\rho^{0}(q) \rightarrow \pi^{+}\left(q_{1}\right) \pi^{+}\left(q_{2}\right) \pi^{-}\left(q_{3}\right) \pi^{-}\left(q_{4}\right)$. One has

$$
\begin{aligned}
J_{\mu}= & \left(1+P_{12}\right)\left(1+P_{34}\right)\left\{-q_{1 \mu}\left[\frac{1}{2}+\frac{2\left(q_{2}, q_{3}\right)}{D_{\pi}\left(q-q_{1}\right)}\right]+q_{3 \mu}\left[\frac{1}{2}+\frac{2\left(q_{1}, q_{4}\right)}{D_{\pi}\left(q-q_{3}\right)}\right]\right. \\
& \left.+\left(2 g_{\rho \pi \pi} f_{\pi}\right)^{2}\left(1+P_{13}\right) \frac{q_{1 \mu}\left(q_{3}, q_{2}-q_{4}\right)}{D_{\pi}\left(q-q_{1}\right) D_{\rho}\left(q_{2}+q_{4}\right)}\right\} .
\end{aligned}
$$

Hereafter $D_{\pi}(q)=m_{\pi}^{2}-q^{2}$ and $D_{\rho}(q)=m_{\rho}^{2}-q^{2}$ are inverse propagators of pion and $\rho$ meson, respectively, and $P_{i j}$ is the operator of interchange of the pion momenta $q_{i}$ and $q_{j}$. Direct numerical evaluation shows that the neglect of the $\rho$ width is justifiable with accuracy better than $5 \%$ up to the energy $\sqrt{s} \leq 0.9 \mathrm{GeV}$. Hereafter $s$ stands for the total center-of-mass energy squared. Recall that the allowing for the finite widths effects is in fact equivalent to the loop correction being taken into account.

2) The decay $\rho^{0}(q) \rightarrow \pi^{+}\left(q_{1}\right) \pi^{-}\left(q_{2}\right) \pi^{0}\left(q_{3}\right) \pi^{0}\left(q_{4}\right)$. In this case one has $J_{\mu}=J_{\mu}^{\text {nan }}+J_{\mu}^{\text {an }}$, where

$$
\begin{aligned}
J_{\mu}^{\text {nan }}= & -\left(1-P_{12}\right)\left(1+P_{34}\right) q_{1 \mu}\left\{\frac{1}{4}+\frac{1}{D_{\pi}\left(q-q_{1}\right)}\left[\left(q_{3}, q_{4}\right)+\left(2 g_{\rho \pi \pi} f_{\pi}\right)^{2} \frac{\left(q_{3}, q_{2}-q_{4}\right)}{D_{\rho}\left(q_{2}+q_{4}\right)}\right]\right\} \\
& +\left(1+P_{34}\right) \frac{\left(g_{\rho \pi \pi} f_{\pi}\right)^{2}}{D_{\rho}\left(q_{1}+q_{3}\right) D_{\rho}\left(q_{2}+q_{4}\right)}\left[\left(q_{1}+q_{3}-q_{2}-q_{4}\right)_{\mu}\left(q_{1}-q_{3}, q_{2}-q_{4}\right)\right. \\
& \left.-2\left(q_{1}-q_{3}\right)_{\mu}\left(q_{1}+q_{3}, q_{2}-q_{4}\right)+2\left(q_{2}-q_{4}\right)_{\mu}\left(q_{2}+q_{4}, q_{1}-q_{3}\right)\right]
\end{aligned}
$$

is obtained from Eq. (1), while the contribution of the term induced by the anomalous lagrangian of Wess and Zumino [6, 10],

$$
\mathcal{L}_{\omega \rho \pi}=\frac{N_{c} g_{\rho \pi \pi}^{2}}{8 \pi^{2} f_{\pi}} \varepsilon_{\mu \nu \lambda \sigma} \partial_{\mu} \omega_{\nu}\left(\boldsymbol{\pi} \cdot \partial_{\lambda} \boldsymbol{\rho}_{\sigma}\right),
$$

manifesting in the process $\rho^{0} \rightarrow \omega \pi^{0} \rightarrow \pi^{+} \pi^{-} \pi^{0} \pi^{0}$, is given by the expression

$$
\begin{aligned}
J_{\mu}^{\text {an }}= & 2\left(\frac{N_{c} g_{\rho \pi \pi}^{2}}{8 \pi^{2}}\right)^{2}\left(1+P_{34}\right)\left[q_{1 \mu}\left(1-P_{23}\right)\left(q, q_{2}\right)\left(q_{3}, q_{4}\right)\right. \\
& \left.+q_{2 \mu}\left(1-P_{13}\right)\left(q, q_{3}\right)\left(q_{1}, q_{4}\right)+q_{3 \mu}\left(1-P_{12}\right)\left(q, q_{1}\right)\left(q_{2}, q_{4}\right)\right] \\
& \times\left[\frac{1}{D_{\rho}\left(q_{1}+q_{2}\right)}+\frac{1}{D_{\rho}\left(q_{1}+q_{3}\right)}+\frac{1}{D_{\rho}\left(q_{2}+q_{3}\right)}\right] \frac{1}{D_{\omega}\left(q-q_{4}\right)},
\end{aligned}
$$

where $D_{\omega}(q)=m_{\omega}^{2}-q^{2}$ is the inverse $\omega$ meson propagator, and $N_{c}=3$ is the number of colors. In general, this term is attributed to the contribution of higher derivatives. Nevertheless, we take it into account to show the effect of the latter and the dynamical effect of the opening of the channel $\rho \rightarrow \omega \pi \rightarrow 4 \pi$. In agreement with [6], the contribution of the point vertex $\omega \rightarrow 3 \pi$ is omitted. The following amplitudes of the charged $\rho$ decay are necessary for obtaining the $\omega \rightarrow 5 \pi$ decay amplitude, and are of their own interest when studying the reactions of peripheral $\rho$ meson production.

3) The decay $\rho^{+}(q) \rightarrow \pi^{+}\left(q_{1}\right) \pi^{0}\left(q_{2}\right) \pi^{0}\left(q_{3}\right) \pi^{0}\left(q_{4}\right)$. One has 


$$
\begin{aligned}
J_{\mu}= & 2 q_{1 \mu}\left[1+\frac{\left(q_{2}, q_{3}\right)+\left(q_{2}, q_{4}\right)+\left(q_{3}, q_{4}\right)}{D_{\pi}\left(q-q_{1}\right)}\right]-\left(1+P_{23}\right) \frac{2 q_{2 \mu}\left(q_{3}, q_{4}\right)}{D_{\pi}\left(q-q_{2}\right)}-\frac{2 q_{4 \mu}\left(q_{2}, q_{3}\right)}{D_{\pi}\left(q-q_{4}\right)} \\
& -\left(2 g_{\rho \pi \pi} f_{\pi}\right)^{2}\left(1+P_{23}\right)\left[\left(1+P_{34}\right) \frac{q_{2 \mu}\left(q_{4}, q_{1}-q_{3}\right)}{D_{\pi}\left(q-q_{2}\right) D_{\rho}\left(q_{1}+q_{3}\right)}+\frac{q_{4 \mu}\left(q_{2}, q_{1}-q_{3}\right)}{D_{\pi}\left(q-q_{4}\right) D_{\rho}\left(q_{1}+q_{3}\right)}\right]
\end{aligned}
$$

4) The decay $\rho^{+}(q) \rightarrow \pi^{+}\left(q_{1}\right) \pi^{+}\left(q_{2}\right) \pi^{-}\left(q_{3}\right) \pi^{0}\left(q_{4}\right)$. Here, the contribution induced by the anomalous lagrangian of Wess and Zumino is also possible, hence $J_{\mu}=J_{\mu}^{\text {nan }}+J_{\mu}^{\text {an }}$, where

$$
\begin{aligned}
J_{\mu}^{\text {nan }}= & \left(1+P_{12}\right)\left\{\left(1-P_{14}\right) q_{1 \mu}\left[\frac{1}{2}+\frac{2\left(q_{2}, q_{3}\right)}{D_{\pi}\left(q-q_{1}\right)}\right]-\left(2 g_{\rho \pi \pi} f_{\pi}\right)^{2}\left[\left(1+P_{23}\right)\right.\right. \\
& \left.\left.\times \frac{q_{1 \mu}\left(q_{2}, q_{3}-q_{4}\right)}{D_{\pi}\left(q-q_{1}\right) D_{\rho}\left(q_{3}+q_{4}\right)}+\frac{q_{4 \mu}\left(q_{1}, q_{2}-q_{3}\right)}{D_{\pi}\left(q-q_{4}\right) D_{\rho}\left(q_{2}+q_{3}\right)}\right]\right\}+\left(g_{\rho \pi \pi} f_{\pi}\right)^{2}\left(1+P_{12}\right) \\
& \times\left\{\left[\left(q_{1}+q_{3}-q_{2}-q_{4}\right)_{\mu}\left(q_{1}-q_{3}, q_{2}-q_{4}\right)-2\left(q_{1}-q_{3}\right)_{\mu}\left(q_{1}+q_{3}, q_{2}-q_{4}\right)\right.\right. \\
& \left.\left.+2\left(q_{2}-q_{4}\right)_{\mu}\left(q_{1}-q_{3}, q_{2}+q_{4}\right)\right] \frac{1}{D_{\rho}\left(q_{1}+q_{3}\right) D_{\rho}\left(q_{2}+q_{4}\right)}\right\}
\end{aligned}
$$

is obtained from Eq. (11), while the term induced by the anomaly looks as

$$
\begin{aligned}
J_{\mu}^{\text {an }}= & 2\left(\frac{N_{c} g_{\rho \pi \pi}^{2}}{8 \pi^{2}}\right)^{2}\left(1+P_{23}\right)\left[q_{1 \mu}\left(1-P_{24}\right)\left(q, q_{4}\right)\left(q_{2}, q_{4}\right)\right. \\
& \left.+q_{2 \mu}\left(1-P_{14}\right)\left(q, q_{1}\right)\left(q_{3}, q_{4}\right)+q_{4 \mu}\left(1-P_{12}\right)\left(q, q_{2}\right)\left(q_{1}, q_{3}\right)\right] \\
& \times\left[\frac{1}{D_{\rho}\left(q_{1}+q_{2}\right)}+\frac{1}{D_{\rho}\left(q_{1}+q_{4}\right)}+\frac{1}{D_{\rho}\left(q_{2}+q_{4}\right)}\right] \frac{1}{D_{\omega}\left(q-q_{3}\right)} .
\end{aligned}
$$

One can verify that up to the corrections of the order of $\sim \mu^{2} / m_{\rho}^{2}$, the above written amplitudes vanish in the limit of vanishing 4-momentum of each final pion. In other words, they obey the Adler condition.

When evaluating the partial widths of the $4 \pi$ decays of $\rho$ meson the modulus squared of the matrix element is expressed via the Kumar variables [12]. The idea of speeding up the numerical integration suggested in Ref. 13] is realized in the numerical algorithm. The results of evaluation of the partial widths at $\sqrt{s}=m_{\rho}=770 \mathrm{MeV}$ are as follows: $\Gamma\left(\rho^{0} \rightarrow\right.$ $\left.2 \pi^{+} 2 \pi^{-}, m_{\rho}^{2}\right)=0.89 \mathrm{keV}, \Gamma\left(\rho^{0} \rightarrow \pi^{+} \pi^{-} 2 \pi^{0}, m_{\rho}^{2}\right)=0.24 \mathrm{keV}$ and $0.44 \mathrm{keV}$, respectively, without and with the induced anomaly term being taken into account. This coincides with the results obtained in 4 . In the case of the charged $\rho$ meson decays it is obtained for the first time: $\Gamma\left(\rho^{+} \rightarrow \pi^{+} 3 \pi^{0}, m_{\rho}^{2}\right)=0.41 \mathrm{keV}, \Gamma\left(\rho^{+} \rightarrow 2 \pi^{+} \pi^{-} \pi^{0}, m_{\rho}^{2}\right)=0.71 \mathrm{keV}$ and $0.90 \mathrm{keV}$ respectively, without and with the anomaly induced term being taken into account.

The results of the $4 \pi$ state production cross section in the reaction $e^{+} e^{-} \rightarrow \rho^{0} \rightarrow 4 \pi$ are shown in Fig. 1 and 2. To demonstrate the effects of chiral dynamics, also shown is the energy dependence of the cross section, which expression contains the quantity $\Gamma^{\mathrm{LIPS}}(s)$ representing the width evaluated in the model of the simple $4 \pi$ phase space normalized to the widths at the $\rho$ mass. When so doing, the ratio $\Gamma\left(\rho \rightarrow 2 \pi^{+} 2 \pi^{-}, s\right) / \Gamma^{\text {LIPS }}\left(\rho \rightarrow 2 \pi^{+} 2 \pi^{-}, s\right)$ changes from 0.4 at $\sqrt{s}=650 \mathrm{MeV}$ to 1 at $\sqrt{s}=m_{\rho}$. As can be observed from the figures, the rise of the $\rho \rightarrow 4 \pi$ partial width with the energy increase is such fast that it compensates completely the falling of the $\rho$ meson propagator and electronic width. Also noticeable is the dynamical effect in the decay $\rho^{0} \rightarrow \pi^{+} \pi^{-} 2 \pi^{0}$ at $\sqrt{s}>850 \mathrm{MeV}$ resulting from the 
anomaly induced lagrangian $\omega \pi$ threshold. See Fig. 2. To quantify the above mentioned effect of vanishing contribution of higher derivatives at the left shoulder of the $\rho$ resonance it should be noted that the difference between magnitudes of $\Gamma\left(\rho \rightarrow \pi^{+} \pi^{-} 2 \pi^{0}, s\right)$ with and without term originating from the anomaly induced lagrangian, equal to $100 \%$ at $\sqrt{s}=m_{\rho}$, diminishes rapidly with the energy decrease amounting to $8 \%$ at $\sqrt{s}=700 \mathrm{MeV}$ and $0.3 \%$ at $\sqrt{s}=650 \mathrm{MeV}$.

As it is seen from Fig. 1, the predictions of chiral symmetry for the $e^{+} e^{-} \rightarrow 2 \pi^{+} 2 \pi^{-}$ reaction cross section do not contradict to the three lowest experimental points of CMD-2 detector [11]. However, at $\sqrt{s}>800 \mathrm{MeV}$ one can observe a substantial deviation between the predictions of the lagrangian (1) the data of CMD-2. In all appearance, this is due to the contribution of higher derivatives and chiral loops neglected in the present work. It is expected that the left shoulder of the $\rho$ is practically free of such contributions, and by this reason it is preferable for studying the dynamical effects of chiral symmetry. Note that even at $\sqrt{s}=650 \mathrm{MeV}$, where the contribution of higher derivatives is negligible, one can hope to gather one event of the reaction $e^{+} e^{-} \rightarrow 2 \pi^{+} 2 \pi^{-}$, provided the luminosity $L=10^{32} \mathrm{~cm}^{-2} \mathrm{~s}^{-1}$ is achieved, and up to 10 events per day at $\sqrt{s}=700 \mathrm{MeV}$, i.e. to have a factory for a comprehensive study of the chiral dynamics of many-pion systems.

One may convince oneself that the $\omega \rightarrow \rho \pi \rightarrow 5 \pi$ decay amplitude unambiguously results from the anomaly induced lagrangian (4). In order to calculate it, notice that, although $\left|\mathbf{q}_{\pi}\right| / m_{\pi} \simeq 0.5$, the nonrelativistic expressions describe the $\rho \rightarrow 4 \pi$ decay widths with the accuracy $10 \%$ in the $4 \pi$ mass range relevant for the present purpose. Using the corresponding limiting expressions for currents (2), (3), (6), (7), and neglecting the corrections of the order of $O\left(\left|\mathbf{q}_{\pi}\right|^{4} / m_{\pi}^{4}\right)$ or higher, one obtains

$$
\begin{aligned}
M\left(\omega \rightarrow 2 \pi^{+} 2 \pi^{-} \pi^{0}\right)= & \frac{N_{c}}{(2 \pi)^{2}}\left(\frac{g_{\rho \pi \pi}}{2 f_{\pi}}\right)^{3} \varepsilon_{\mu \nu \lambda \sigma} q_{\mu} \epsilon_{\nu}\left[\left(1+P_{12}\right) \frac{q_{1 \lambda}\left(q_{2}+3 q_{4}\right)_{\sigma}}{D_{\rho}\left(q-q_{1}\right)}\right. \\
& \left.-\left(1+P_{35}\right) \frac{q_{3 \lambda}\left(q_{5}+3 q_{4}\right)_{\sigma}}{D_{\rho}\left(q-q_{3}\right)}+4 \frac{q_{4 \lambda}\left(q_{3}+q_{5}\right)_{\sigma}}{D_{\rho}\left(q-q_{4}\right)}\right]
\end{aligned}
$$

with the final momentum assignment according to $\pi^{+}\left(q_{1}\right) \pi^{+}\left(q_{2}\right) \pi^{-}\left(q_{3}\right) \pi^{-}\left(q_{5}\right) \pi^{0}\left(q_{4}\right)$, and

$$
\begin{aligned}
M\left(\omega \rightarrow \pi^{+} \pi^{-} 3 \pi^{0}\right)= & \frac{N_{c}}{(2 \pi)^{2}}\left(\frac{g_{\rho \pi \pi}}{2 f_{\pi}}\right)^{3} \varepsilon_{\mu \nu \lambda \sigma} q_{\mu} \epsilon_{\nu}\left\{\left(1-P_{12}\right) \frac{4 q_{2 \lambda} q_{1 \sigma}}{D_{\rho}\left(q-q_{1}\right)}\right. \\
& \left.+\left(q_{1}-q_{2}\right)_{\lambda}\left[\frac{q_{3 \sigma}}{D_{\rho}\left(q-q_{3}\right)}+\frac{q_{4 \sigma}}{D_{\rho}\left(q-q_{4}\right)}+\frac{q_{5 \sigma}}{D_{\rho}\left(q-q_{5}\right)},\right]\right\} .
\end{aligned}
$$

with the final momentum assignment according to $\pi^{+}\left(q_{1}\right) \pi^{-}\left(q_{2}\right) \pi^{0}\left(q_{3}\right) \pi^{0}\left(q_{4}\right) \pi^{0}\left(q_{5}\right)$. In both above formulas, $\epsilon_{\nu}, q_{\mu}$ stand for four-vectors of polarization and momentum of $\omega$ meson.

Since the invariant mass of the $4 \pi$ system changes in very narrow range $558 \mathrm{MeV}<$ $m_{4 \pi}<642 \mathrm{MeV}$, one can set it in all the $\rho$ propagators, with the accuracy $20 \%$ in width, to the equilibrium value $\bar{m}_{4 \pi}=620 \mathrm{MeV}$ evaluated for the pion energy $E_{\pi}=m_{\omega} / 5$ which gives the dominant contribution. The resulting $\omega \rightarrow 2 \pi^{+} 2 \pi^{-} \pi^{0}$ and $\omega \rightarrow \pi^{+} \pi^{-} 3 \pi^{0}$ decay amplitudes equal to $5 / 2$ times the $\omega \rightarrow \rho^{0} \pi^{0} \rightarrow 2 \pi^{+} 2 \pi^{-} \pi^{0}$ and $\omega \rightarrow \rho^{+} \pi^{-} \rightarrow \pi^{+} \pi^{-} 3 \pi^{0}$ decay amplitudes, respectively.

It would be useful to fulfill the model estimate of the $\omega \rightarrow 5 \pi$ partial widths as follows. Corresponding pion momenta are $\left|\mathbf{q}_{\pi^{+}}\right|=70 \mathrm{MeV}$ and $\left|\mathbf{q}_{\pi^{0}}\right|=79 \mathrm{MeV}$. The integrations over 
angles of final pions can be fulfilled assuming them independent. Using the nonrelativistic expression for phase space of five pions [14],

$$
R_{5}=\frac{\pi^{6}\left(\prod_{i}^{5} m_{\pi i}\right)^{1 / 2}}{60\left(\sum_{i}^{5} m_{\pi i}\right)^{3 / 2}}\left(m_{\omega}-\sum_{i}^{5} m_{\pi i}\right)^{5}
$$

whose numerical value coincides with the accuracy $1 \%$ with the numerically evaluated exact expression, one finds

$$
\begin{aligned}
B(\omega \rightarrow 5 \pi) \simeq & {\left[\frac{5 N_{c}}{2 \pi^{2}}\left(\frac{g_{\rho \pi \pi}}{2 f_{\pi}}\right)^{3} \frac{m_{\omega}\left|\mathbf{q}_{\pi^{+}}\right|}{m_{\rho}^{2}-\bar{m}_{4 \pi}^{2}}\right]^{2} } \\
& \times \frac{R_{5}}{18(2 \pi)^{11} m_{\omega} \Gamma_{\omega}} \\
& \times\left\{\begin{array}{l}
\left|\mathbf{q}_{\pi^{0}}\right|^{2}\left(2 \pi^{+} 2 \pi^{-} \pi^{0}\right) \\
\frac{\left|\mathbf{q}_{\pi^{+}}\right|^{2}}{3}\left(\pi^{+} \pi^{-} 3 \pi^{0}\right)
\end{array}\right.
\end{aligned}
$$

The calculation gives $B\left(\omega \rightarrow 2 \pi^{+} 2 \pi^{-} \pi^{0}\right)=4.1 \times 10^{-9}$ and $B\left(\omega \rightarrow \pi^{+} \pi^{-} 3 \pi^{0}\right)=1.7 \times 10^{-9}$.

The evaluation of the partial widths valid with accuracy $20 \%$ can be obtained upon taking the amplitude of each considered decays as $5 / 2$ times the $\rho \pi$ production state amplitude with the subsequent decay $\rho \rightarrow 4 \pi$, and calculate the partial width using the calculated widths of the latter:

$$
\begin{aligned}
B_{\omega \rightarrow 2 \pi^{+} 2 \pi^{-} \pi^{0}}= & \left(\frac{5}{2}\right)^{2} \frac{2}{\pi \Gamma_{\omega}} \int_{4 m_{\pi^{+}}}^{m_{\omega}-m_{\pi^{0}}} d m \\
& \times \frac{m^{2} \Gamma_{\omega \rightarrow \rho^{0} \pi^{0}}(m) \Gamma_{\rho \rightarrow 2 \pi^{+} 2 \pi^{-}}(m)}{\left|D_{\rho}\left(m^{2}\right)\right|^{2}} \\
& =1.6 \times 10^{-9}
\end{aligned}
$$

$\Gamma_{\omega \rightarrow \rho^{0} \pi^{0}}(m)=g_{\omega \rho \pi}^{2} q^{3}\left(m_{\omega}, m, m_{\pi^{0}}\right) / 12 \pi, g_{\omega \rho \pi}=N_{c} g_{\rho \pi \pi}^{2} / 8 \pi^{2} f_{\pi}=14.3 \mathrm{GeV}^{-1}$, and $q\left(m_{a}, m_{b}, m_{c}\right)$ is the momentum of final particle $b$ (or $c$ ) in the rest frame system of decaying particle $a$. The partial width $B_{\omega \rightarrow \pi^{+} \pi^{-} 3 \pi^{0}}=1.2 \times 10^{-9}$ is obtained from Eq. (13) upon inserting the lower integration limit to $m_{\pi^{+}}+3 m_{\pi^{0}}$, the upper one to $m_{\omega}-m_{\pi^{0}}$, and $m_{\pi^{0}} \rightarrow m_{\pi^{+}}$in the expression for the momentum $q$ and substitution of the $\rho^{+} \rightarrow \pi^{+} 3 \pi^{0}$ decay width corrected for the mass difference of charged and neutral pions. Provided the luminosity $L=10^{33} \mathrm{~cm}^{-2} \mathrm{~s}^{-1}$ is achieved, one may expect about 3 events per week for the considered decays.

We are grateful to A. E. Bondar and A. I. Sukhanov for the kindly supplied table of experimental data and discussion. The present work is supported in part by the grant RFBR-INTAS IR-97-232. 


\section{REFERENCES}

[1] L. Bányai and V. Rittenberg, Phys. Rev. 184, 1903 (1969).

[2] A. Bramon, A. Grau, and G. Pancheri, Phys. Lett. B317, 190 (1993).

[3] S. I. Eidelman, Z. K. Silagadze, and E. A. Kuraev, Phys. Lett. B346, 186 (1995).

[4] R. S. Plant and M. C. Birse, Phys. Lett. B365, 292 (1996).

[5] S. Weinberg, Phys. Rev. 166, 1568 (1968).

[6] M. Bando, T. Kugo, S. Uehar et al., Phys. Rev. Lett. 54, 1215 (1985); M. Bando, T. Kugo, and K. Yamawaki, Nucl. Phys. B259, 493 (1985); Progr. Theor. Phys. 73, 1541 (1985); Phys. Rep. 164, 217 (1988).

[7] The problem of inclusion of vector, axial mesons, and photons to the framework of chiral theories has demanded considerable efforts. See J. Schwinger, Phys. Lett. 24B, 473 (1967); J. Wess and B. B. Zumino, Phys. Rev. 163, 1727 (1967); S. Gasiorowicz and D. A. Geffen, Rev. Mod. Phys. 41, 531 (1969); O. Kaymakcalan, S. Rajeev and J. Schechter, Phys. Rev. D30, 594 (1984); U. -G. Meissner, Phys. Rep. 161, 213 (1988), and is solved in an elegant way in the approach based on hidden local symmetry [6].

[8] Taking the higher derivatives into account demands also taking into account the chiral loops, the task which is not yet fulfilled for vector mesons.

[9] K. Kawarabayashi and M. Suzuki, Phys. Rev. Lett. 16, 255 (1966);

Riazzuddin and Fayyazuddin, Phys. Rev. 147, 1071 (1966).

[10] J. Wess and B. B. Zumino, Phys. Lett. 37B, 95 (1971).

[11] R. R. Akhmetshin et al., Novosibirsk preprint BINP 99-11 (1999).

[12] R. Kumar, Phys. Rev. 185, 1865 (1969).

[13] T. W. Sag and G. Szekeres, Math. Comput. 18, 245 (1964).

[14] E. Byckling and K. Kajantie, Particle Kinematics (John Wiley and Sons, London-New York-Sydney-Toronto, 1973) ch. VI. 


\section{FIGURES}

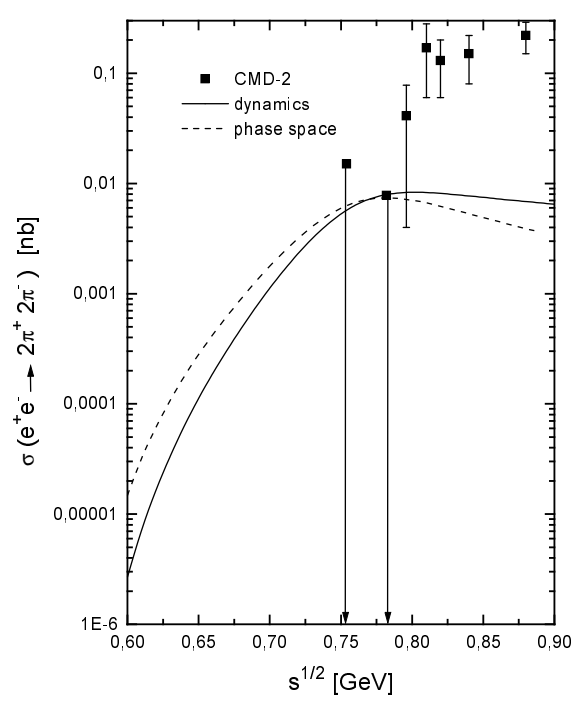

FIG. 1. The energy dependence of the $e^{+} e^{-} \rightarrow \rho^{0} \rightarrow \pi^{+} \pi^{-} \pi^{+} \pi^{-}$reaction cross section in the model based on the chiral lagrangian due to Weinberg, Experimental points are from [11.

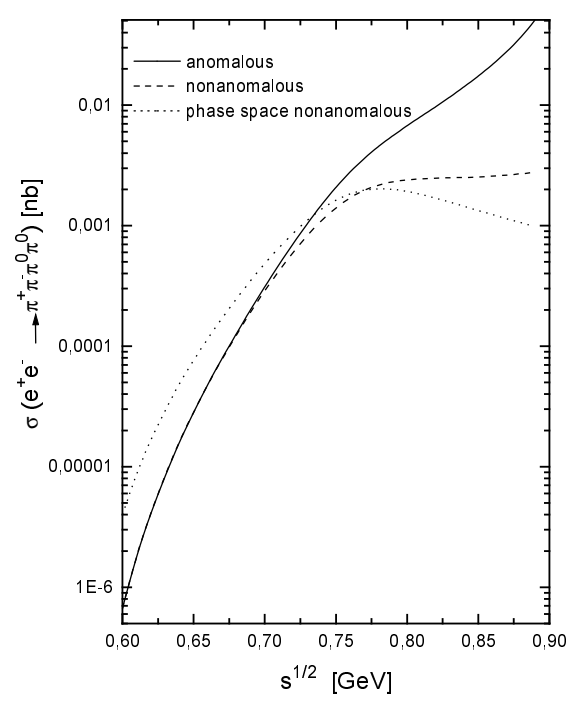

FIG. 2. The energy dependence of the $e^{+} e^{-} \rightarrow \rho^{0} \rightarrow \pi^{+} \pi^{-} \pi^{0} \pi^{0}$ reaction cross section in the model based on the chiral lagrangian due to Weinberg. 